\title{
AUTOIMMUNE MYELOFIBROSIS IN SYSTEMIC LUPUS ERYTHEMATOUS: A CASE SERIES
}

Izabela Zarnowski Passos ${ }^{1, *}$, Ana Maria São Thiago Santiago Bez ${ }^{1}$, Christiane Cota Vieira ${ }^{1}$, Deborah Lobato Guimarães ${ }^{1}$, Diego Leles Ribeiro ${ }^{1}$, Lucas Leonardo de Castro Borges ${ }^{1}$, Luiz Severiano Ribeiro ${ }^{1}$, Maria Fernanda Pereira Moreira ${ }^{1}$, Maria Letícia Pinheiro Campos², Rejane Pinheiro Damasceno ${ }^{1}$

1.Hospital Governador Israel Pinheiro, Belo Horizonte (MG), Brazil. 2. Faculdade Ciências Médicas de Minas Gerais, Belo Horizonte (MG), Brazil. *Corresponding author: izabelazarnowskipassos@gmail.com

\section{BACKGROUND}

Autoimmune myelofibrosis is an uncommon event among patients with systemic lupus erythematous (SLE) and should always be suspected in the presence of pancytopenia. In a review of the literature until 2018, 45 cases of this association were described. We report three cases that were evaluated in our service, compiled in the last 26 years. In one of the cases there was a history of exposure to myelotoxic drugs; in the remaining two cases, the diagnosis was made concomitantly with that of SLE.

\section{CASE REPORT}

Case 1: 20-year-old female diagnosed with SLE at 13 years old, with a previous history of exposure to intravenous (IV) cyclophosphamide, azathioprine and mycophenolate mofetil. She had developed an end-stage renal disease (ESRD) and was hospitalized with pancytopenia, fever, hypotension, ascites and abdominal pain, with a SELENA-SLEDAI 9. Bone marrow biopsy was performed and showed hypocellular medulla, mainly in the granulocytic series, associated with Bauermeister grade 2 fibrosis. The patient underwent systemic immunosuppressive therapy with corticosteroids, immunoglobulin and rituximab, without response to the treatment, evolving to death due to nosocomial infection. Case 2: 34-year-old female, admitted with daily vespertine fever; cervical and occipital lymphadenomegaly and hepatomegaly, diagnosed with SLE during hospitalization, with SELENA-SLEDAI 15. Myelogram was performed resulting in a dry aspiration and bone marrow biopsy showing a moderately hypocellular medulla and presence of reticulin fibrosis. She was treated with systemic corticosteroid therapy and IV cyclophosphamide, and, in the concomitance of nephritis, evolved with complete remission. Case 3: 24-year-old female, hospitalized with fever, myalgia, photosensitive skin rash on the face and pancytopenia, fulfilling classification criteria for SLE, with a SELENA-SLEDAI 13. The myelogram was hypocellular, and the bone marrow biopsy evidenced focal reticulin fibrosis. The patient was treated with prednisone and chloroquine diphosphate, with improvement of symptoms and normalization of hematologic alterations.

\section{CONCLUSION}

Hematologic manifestations are varied and commonly found in SLE as a presenting feature or during the course of the disease. Autoimmune myelofibrosis, however, is an uncommon finding in SLE. It should be suspected in the presence of pancytopenia and confirmed by myelogram and bone marrow biopsy evidencing fibrosis. This condition may be reversible with immunosuppressive therapy, but a patient with ESRD, under high-grade immunosuppression, developed a serious infection, resulting in her death. 